\title{
PASSIVE ISOLATORS FOR USE ON THE INTERNATIONAL SPACE STATION
}

\author{
J. Houston \\ Jacobs Sverdrup Technology, Inc. \\ Huntsville, AL 806 \\ Janice.Houston@msfc.nasa.gov \\ Christy B. Gattis \\ NASA Marshall Space Flight Center \\ Huntsville, AL 35812 \\ christy.gattis@msfc.nasa.gov
}

\begin{abstract}
The value of the International Space Station (ISS) as a premier microgravity environment is currently at risk due to structure-borne vibration. The vibration sources are varied and include crew activities such as exercising or simply moving from module to module, and electromechanical equipment such as fans and pumps. Given such potential degradation of usable microgravity, anything that can be done to dampen vibration on-orbit will significantly benefit microgravity users. Most vibration isolation schemes, both active and passive, have proven to be expensive - both operationally and from the cost of integrating isolation systems into primary/secondary structural interfaces (e.g., the ISS module/rack interface). Recently, passively absorptive materials have been tested at the bolt interfaces between the operating equipment and support structure (secondary/tertiary structural interfaces). The results indicate that these materials may prove cost-effective in mitigating the vibrational problems of the ISS.

We report herein tests of passive absorbers placed at the interface of a vibration-inducing component: the Development Distillation Assembly, a subassembly of the Urine Processing Assembly, which is a rotating centrifuge and cylinder assembly attached to a mounting plate. Passive isolators were installed between this mounting plate and its support shelf. Three materials were tested: BISCO HT -800 , Sorbothane 30 and Sorbothane 50 , plus a control test with a hard shim. In addition, four distinct combinations of the HT-800 and Sorbothane 50 were tested. Results show a significant (three orders of magnitude) reduction of transmitted energy, as measured in power spectral density (PSD), using the isolation materials. It is noted, however, that passive materials cannot prevent the transmission of very strong forces or absorb the total energy induced from structural resonances.
\end{abstract}

INTRODUCTION

A primary mission of the ISS Program is to provide a premier microgravity laboratory environment for fluids, combustion, materials, biotechnology, fundamental physics, and life science research. ${ }^{1}$ In order to address this mission, the ISS needs a structured and effective process to efficiently implement microgravity requirements. The SSP 50036 "Microgravity Control Plan" is the binding document that defines the ISS process for the implementation of the microgravity requirements.

In addition to the "Microgravity Control Plan", unique specification documents exist for the ISS, with SSP 41000 "System Specification for the International Space Station" as the top-level requirements document which flows down requirements to elements and modules which are the ISS components. The Node 3 is an ISS module which houses systems equipment to perform the following functions: distribute and transfer commands, data, audio, video, electrical power, atmosphere, water, and thermal energy to adjacent ISS elements; generate oxygen; process waste water and urine; provide for food storage, consumption, and cleanup; and provide for crewmember cleansing and trash containment. The Node 3 does not house any payloads. The Node 3 microgravity requirement for vibratory accelerations is given in SSP 50318 "Prime Item Specification for Node 3" and shown in Figure 1 on a linear scale. ${ }^{2}$

The Node 3 is comprised of several racks, midbay racks, and alcove racks all of which house equipment that is necessary to maintain electrical power, data handling, thermal, etc. While there is an overall microgravity requirement for the Node 3 as an integrated element, the microgravity requirement is not flowed down to its subsystems or individual racks. We refer to this as a requirements flowdown breakdown. A 
majority of subsystem racks are designed and built by several subcontractors; not the element integrator.

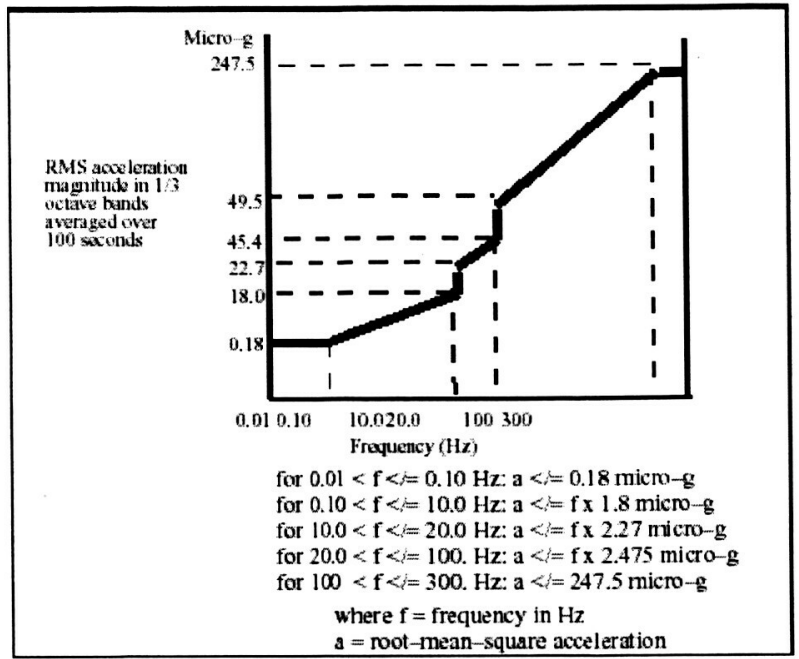

Figure 1. Node 3 Vibratory Microgravity Requirement.

The consequence is that the element integrator becomes responsible for the microgravity performance of the entire Node 3 while not having control over the design and implementation of the individual racks or individual equipment. Thus, the microgravity requirement is not design driven, but rather performance driven.

A performance requirement is difficult to build to and verify. When a problem is identified, (i.e. an exceedance of the requirement), it is found at the top system level, after all the subsystem level equipment has been designed and built. A solution to help the integrator would have been to suballocate the microgravity allotment to each rack so then the rack provider would have been responsible for adhering to its rack microgravity requirement. However, suballocation was not performed for the Node 3 and this should be considered a systems engineering oversight. In addition, the rack and/or equipment providers are not required to test or characterize the microgravity disturbers.

As such, a great deal of guess work is necessary on the part of the integrator to describe the forcing functions for the microgravity disturbers. Vibratory microgravity disturbers have force or sound power level responses in the low frequencies between 0.01 and $300 \mathrm{~Hz}$. Typical microgravity disturbers in this frequency range are the mechanical operation of pumps, fans, valves, and vents and acoustic noise from sources such as fans, blowers, duct inlets and outlets, and pumps. Rotating equipment and motor imbalance also affect the microgravity environment at low frequencies.
Analytical assessments are performed to determine compliance with the microgravity specification. Typically, microgravity testing is limited since the verification method is by analyses. However, if the analytical assessment shows an exceedance of the microgravity requirement, a test will be performed to determine if the analysis overpredicts the microgravity environment. It is common for microgravity testing to occur at the element level, when it is typically too late to implement design changes, such as anti-vibration mounts.

In the case for the Node 3, the analytical predictions proved that a specific rack, Water Recovery System \#2 (WRS\#2) was exceeding the microgravity requirement and testing was performed in the Spring of $1999 .{ }^{3}$

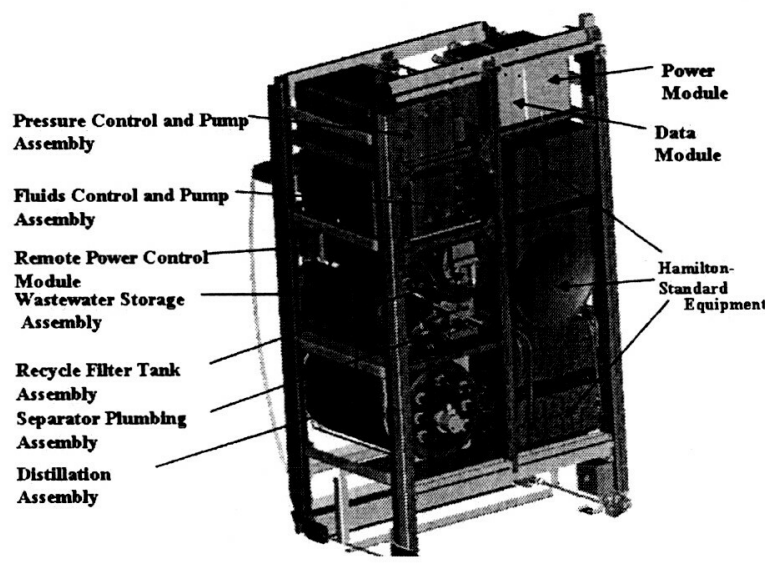

Figure 2. Water Recovery System \#2 for the Node 3.

The testing focused on an orbital replacement unit (ORU) in the WRS\#2 called the Urine Processing Assembly (UPA). The UPA has three components with moving parts that are classified as microgravity disturbers: Distillation Assembly (DA), Fluids Control \& Pump Assembly (FCPA), and Pressure Control \& Pump Assembly (PCPA). The test measured accelerometer data of all three components operating simultaneously on a tabletop. Overall, this test was not representative of the flight configuration, but the primary goal was to determine if the analytical forcing function was an overprediction. Using this test data, it showed that the UPA components were using a majority of the allocation as can be seen in Figure 3 (plotted in log-log format). ${ }^{4}$ An item is considered a Microgravity Critical Item if the accelerations induced by its operations are equal to or greater than 50 percent of the element requirement. ${ }^{1}$ The WRS\#2, which houses the UPA, is a Microgravity Critical Item for the Node 3.

Due to the test setup, there were questions regarding the individual forcing function for each component. As 
such, the UPA team was amenable to retesting the microgravity disturbers separately under flight-like boundary conditions to determine the real microgravity performance of the individual components.

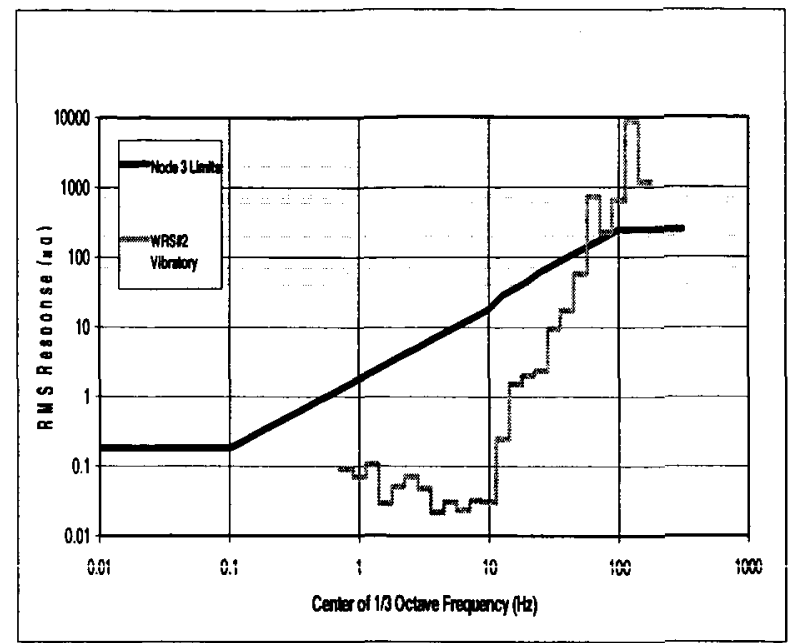

Figure 3. Node 3 WRS\#2 microgravity results compared to Node 3 microgravity requirement (Spring 1999).

The FCPA and PCPA were tested separately in Fall 2000. All results presented are plotted in log-log format. The results of the WRS\#2 Rack compared to the Node 3 microgravity requirement with the new FCPA and PCPA data, shown in Figure 4, were presented at the Critical Design Review (CDR) in Spring 2002. ${ }^{5}$ The results do show an improvement compared to the first test data set shown in Figure 3, specifically in the higher frequencies. Lower frequency results are due to new data at lower frequencies.

The Distillation Assembly hardware was not available for test until post-CDR. The updated Node 3 WRS\#2 results, with the new FCPA, PCPA and DA test data, are shown in Figure 5. ${ }^{6}$ Comparison of Figures 4 and 5 show that the new DA data does not significantly reduce the overall WRS\#2 microgravity environment results.

Since the microgravity requirement was still exceeded even with new and improved test results based upon new testing conditions, and since the results proved conclusively that the WRS\#2 rack is a Microgravity Critical Item, it was decided to implement a design change. The goal was to isolate the component from its shelf structure with an inexpensive grommet. ${ }^{7}$ Since the hardware was already built, this became a design driven solution.

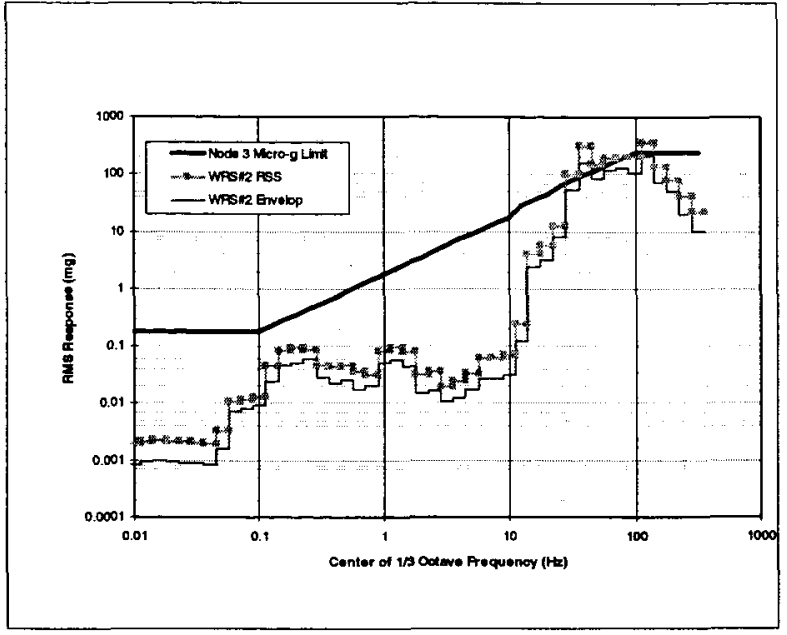

Figure 4. Node 3 WRS\#2 microgravity results with updated FCPA and PCPA data (Fall 2000).

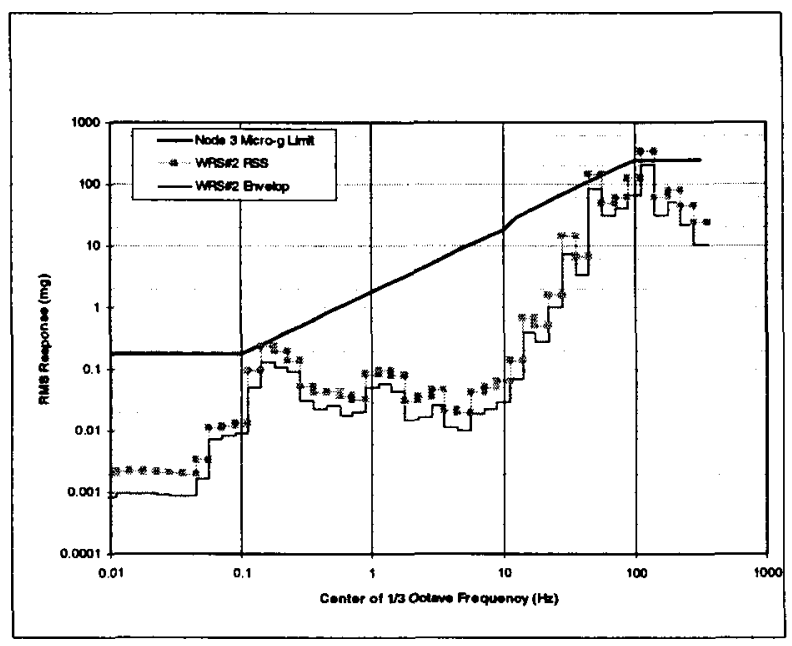

Figure 5. Node 3 WRS\#2 microgravity results with updated FCPA, PCPA and DA data (Spring 2002).

\section{ISOLATORS}

When designing payloads, i.e. experiments, for microgravity research aboard ISS, the issue of vibration isolation is usually at the forefront in design studies. This parameter would typically drive the design toward either low-vibration components or isolation, either passive or active, where necessary to reduce the vibration output. In this case, however, the payload is actually a portion of the regenerative Environmental Control and Life Support System (ECLSS) - a system, not an experiment. For that reason, along with lack of requirements flowdown, the vibration produced by the components within the rack was not considered in the 
early design. Consequently, an "add-on" type of passive isolation was needed.

Ideally, many parameters are considered when choosing an isolator. First, the transmissibility of the material must be studied with respect to the frequency spectrum of the vibration. Vibration will be directly transmitted or amplified by an isolator material up to its resonant peak frequency and then will be attenuated above that frequency. This transmissibility will vary from material to material, and will also vary with hardness (durometer) for a given material. Second, material properties, such as outgassing, toxicity, etc. must also be verified to be compatible with ISS requirements. Last, the physical properties of the material must be studied as well. Elastomeric materials are generally very non-linear with respect to compression versus performance, as well as hysteresis.

Typically, performance of several types of isolators, in conjunction with the mounting provisions of the component to be isolated, should be traded to determine the best design solution for the application. There are many types of isolator solutions available, such as mechanical (springs, dampers), active (controlled piezoelectrics, voice coil actuators), and passive (elastomeric pads, bushings). However, in this case of the UPA components, the isolators had to be introduced into the existing design envelope, which allowed for only a 0.5 " thick by 1 " diameter footprint. With size and shape as the driving factors, the options were drastically reduced, leaving an elastomeric washer and bushing as the only feasible choice. Figure 6 shows a sketch of the isolator bolt for the Distillation Assembly.

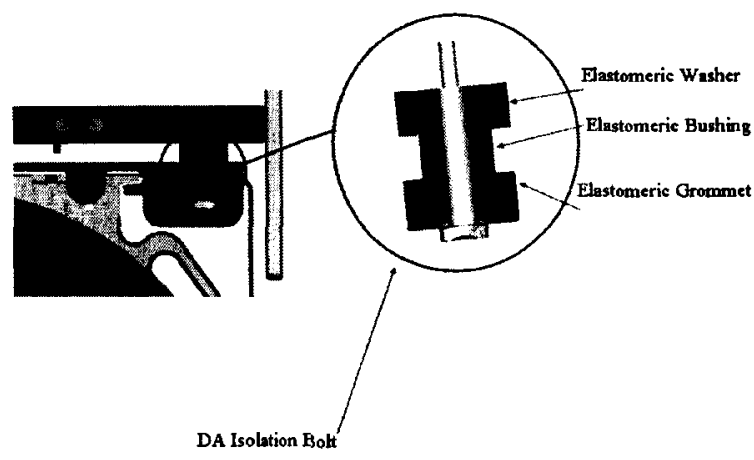

Figure 6. Sketch of the Distillation Assembly bolt with isolator material.
When choosing the specific type of elastomeric isolator, many parameters were considered: transmissibility, materials properties, and physical properties. Because elastomers tend to behave nonlinearly, manufacturers typically recommend some defined preload for these materials. In general, most commercial applications of elastomeric isolators are low-frequency, high-mass vibration sources, such as large machine tools. For these applications, it has been necessary for manufacturers to provide only characterization (i.e. transmissibility data) for these materials with a large preload.

It is here that the problem becomes more complicated by the unique environment of the ISS. The components are hard-mounted (bolted) to rack shelves for launch to the ISS. However, while on-orbit the only required constraint is for physical restraint of the component not load carrying. To accommodate this, the launch bolts will be removed once on-orbit, isolators put in place, and bolts replaced only hand-tight. The compression on the elastomer from the bolts is thus removed, and the elastomer response is in the nonlinear range. Because of this unique situation, it was determined that configuration-specific testing was required to characterize the effectiveness of this isolator design.

Upon investigating several materials as potential candidates for this application, two were determined to be worthy of application-specific testing. The first material was Sorbothane, a polyurethane elastomer, of both 30 and 50 durometer (Shore 00 scale). Two sets of isolators (washer and grommet) and spares were custom-molded by the manufacturer, Sorbothane, Inc. for each of the hardnesses. The other candidate material was BISCO HT- 800 , manufactured by Rogers Corporation. HT-800 is an open-cell silicone designed primarily for use in acoustic isolation. These isolators were hand cut with dies from sheets of material.

\section{ISOLATOR TESTING}

The Node 3 Development Distillation Assembly Microgravity Isolator Materials Characterization Test is a qualitative test. The test objective was to acquire acceleration measurements (power spectral densities (PSDs)) of the DA with different isolators at the boundary conditions while in a simulated zero-g condition. The analytical goal was to compare the relative performance characteristics of the isolators and determine which will provide the best isolation between the DA and the shelf.

There is one analytical limitation due to the data: a forcing function cannot be calculated because no transfer functions were acquired. 
The test occurred at Marshall Space Flight (MSFC) Building 4619 in August 2002. The DA was suspended from a bungee as seen in Figure 7. Most of the hardware tested was flight-like including the DA, mounting plate, and shelf. Mass simulators were built for the Waste Water Storage Tank Assembly (WSTA) and Separator Plumbing Assembly (SPA) and attached to a shelf as seen in Figure 8 . The shelf and mounting plate were attached to the massive test fixture, which was verified to be nonresponsive below $200 \mathrm{~Hz}$.

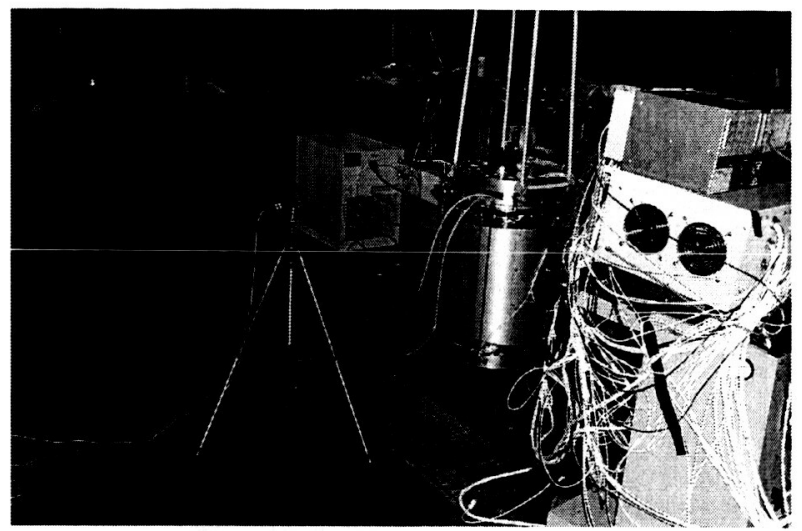

Figure 7. Distillation Assembly test setup.

\section{Test Isolators}

Three materials were tested: Sorbothane 30, Sorbothane 50, and BISCO HT-800. Hard shims were used as a control case. The Sorbothane 30 and 50 isolators were flight-like and were molded to the proper dimensional size by the manufacturer. The hard shims were fabricated in-house. However, the BISCO HT-800 isolators were not flight like, with the tops and bushing pieces separated and cut by hand.

\section{$\underline{\text { Non-Flight Conditions }}$}

There were other non-flight like conditions. The isolators were installed at four bolt interfaces instead of the onorbit configuration of six bolt interfaces. The ground support equipment and operation of the DA were not flight-like. The DA motor was $120 \mathrm{VDC}$ rather than the flight 28 VDC. In addition, the DA operation did not include cooling, fluids, or purging. Overall, these conditions do not change the structural dynamics significantly, which are dominated by the motor and centrifuge harmonics.

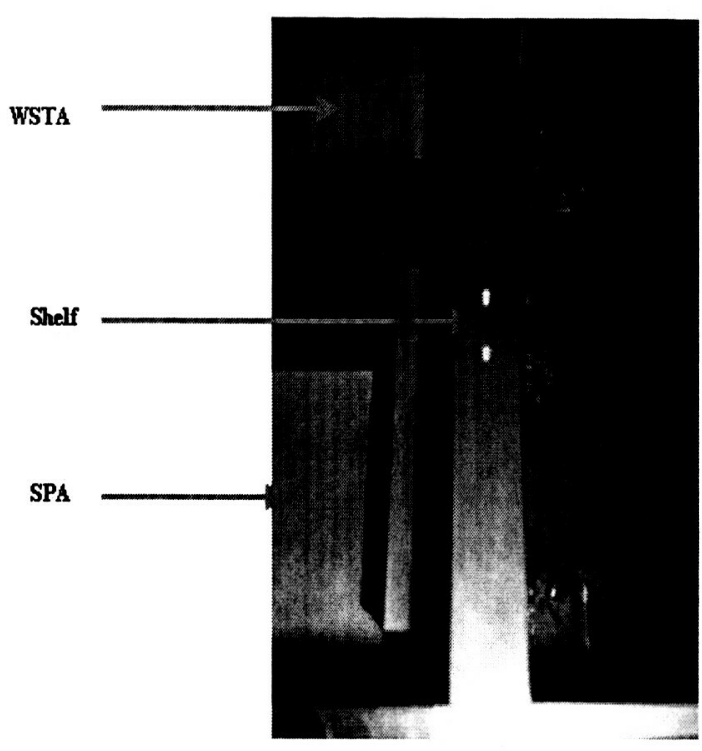

Figure 8. Top down view showing mass simulators.

\section{$\underline{\text { Test Instrumentation }}$}

Acceleration data was acquired during the operation of the DA. The frequency band for the data collection is $0.125 \mathrm{~Hz}-512 \mathrm{~Hz}$ but the frequency range of interest extends only to $350 \mathrm{~Hz}$. There were eight tri-axial accelerometers, four on the non-isolated side and four on the isolated side as seen in Figure 9. A close-up of an isolated and non-isolated tri-axial accelerometer pair is shown in Figure 10.

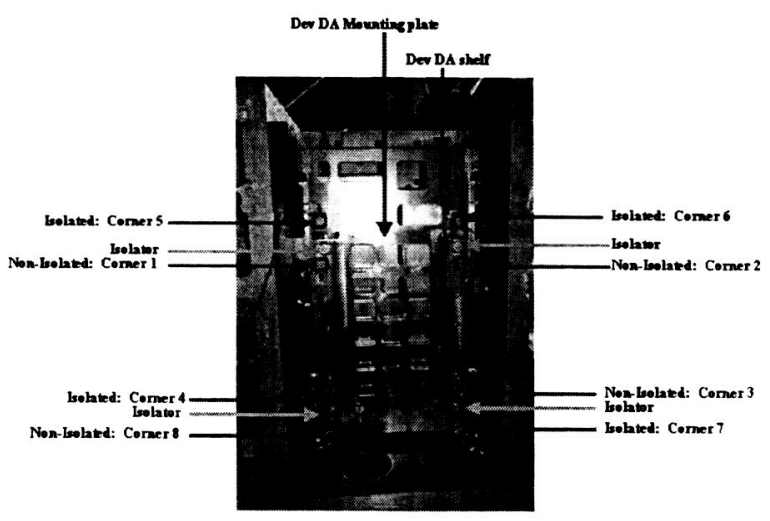

Figure 9. Photo showing location of all isolated and non-isolated accelerometers. 


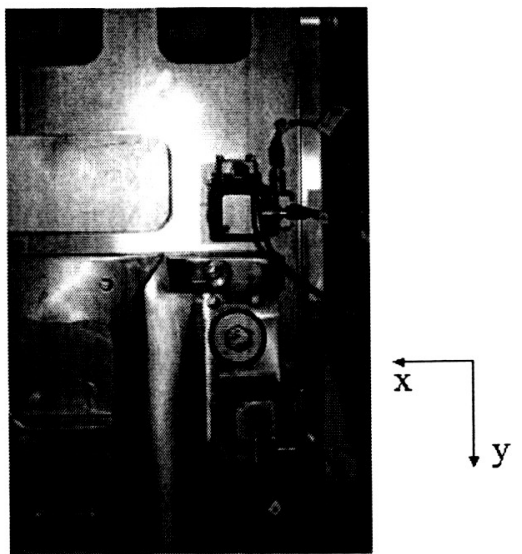

Figure 10. Close up of isolated/non-isolated accelerometer pair with close-up of isolator.

\section{Real-time Change to Test Parameters}

Preliminary quick-look at the data during testing showed that the BISCO HT-800 and Sorbothane 50 materials were the best isolators. There was speculation as to whether combining the two materials would yield an improved isolator. One data combination data set was acquired but each corner was outfitted with a separate and distinct isolator-combo to test as many combinations as possible. The combinations per location are shown in Figure 11. The BISCO-HT800 material was "reused" from the first run since there was not enough material for a second set. However, all of the Sorbothane 50 pieces were new for the combination test case.

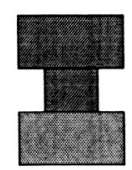

Comers 1 and $S$

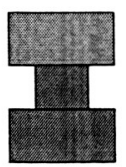

Comers 4 and 8

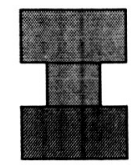

Comers 2 and 6

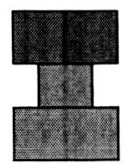

Comers 5 and $?$.

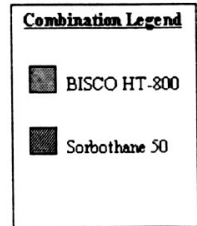

Figure 11. All combinations of isolators per location.

\section{RESULTS}

The first question of importance is whether the operational signal of the Distillation Assembly was above the noise floor. Figure 12 shows the noise floor data in the $\mathrm{z}$-direction. ${ }^{8}$ Compared to the worst case scenario, the DA operating with hard shims as seen in Figure 13, it is apparent that there is significant difference between the noise floor and the operating condition of the DA. ${ }^{8}$ Figure 14 shows the results of the BISCO HT- 800 with the response of the isolated set of accelerometers significantly lower than the response of the non-isolated set of accelerometers.

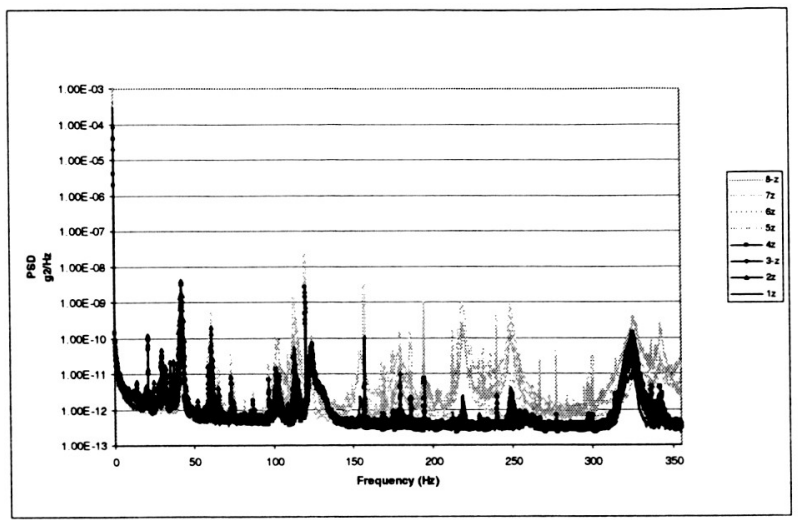

Figure 12. Noise floor in $\mathrm{z}$ direction.

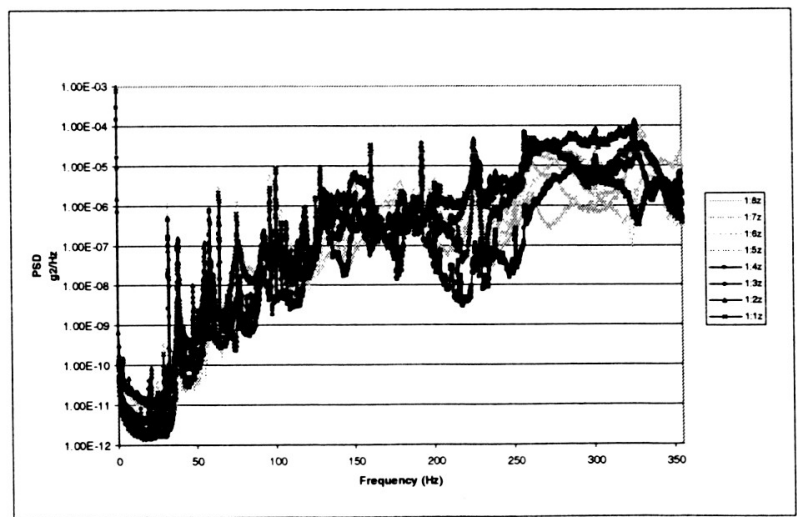

Figure 13. Worst case signal in $\mathrm{z}$ direction with hard shims.

Close examination of the isolator responses in Figure 15 show that the BISCO HT- 800 is the best isolator for most of the frequency range of interest. The BISCO HT-800 response, in gray, is almost equal to the noise floor data from $0-50 \mathrm{~Hz}$ and after $50 \mathrm{~Hz}$ is lower than the other responses for a majority of the frequency range. 


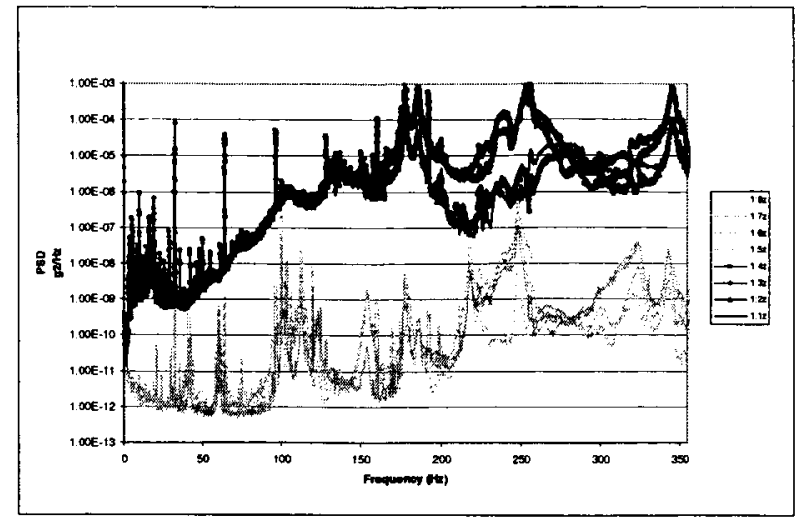

Figure 14. HT-800 BISCO data comparing isolated and non-isolated accelerometer data.

Figure 16 shows the comparison of the noise floor, the BISCO HT-800, and the hard shim case. ${ }^{8}$ As can be seen, the BISCO HT- 800 is three orders of magnitude lower than the control hard shim response in several frequency ranges. The data conclusively shows that the BISCO HT-800 is an effective isolator across the frequency range of interest.

\section{CONCLUSION}

In conclusion, the Distillation Assembly Microgravity Isolator Materials Characterization Test successfully shows that passive isolation works. For the low frequency vibration problem, BISCO HT-800 was the best performing material when compared to Sorbothane 30 and 50. Overall, BISCO HT- 800 is low cost and fits the design parameters. The design has been implemented for the UPA components, the DA, FCPA, and PCPA, and will be in place for on-orbit flight.

Future work will include acquiring forcing functions of the DA, FCPA, and PCPA with isolators in the test setup. Using these isolated forcing functions, the Node 3 microgravity environment due to the WRS\#2 will be calculated to see if the Node 2 microgravity requirement is met. In addition, a survey of isolator materials planned for use by different ISS users is of great interest and will be conducted.

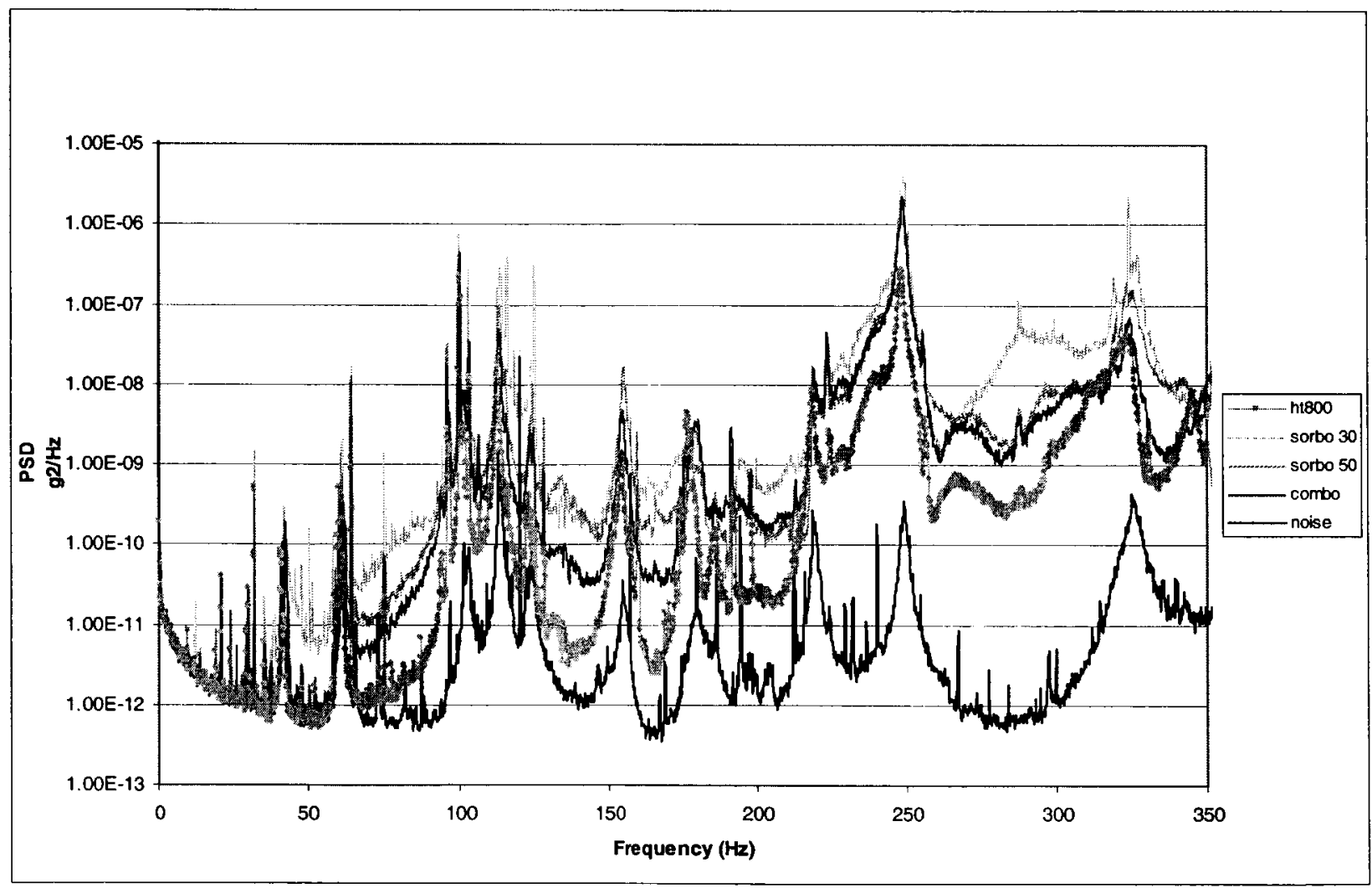

Figure 15. Comparison of all isolators at location $7 \mathrm{z}$. 


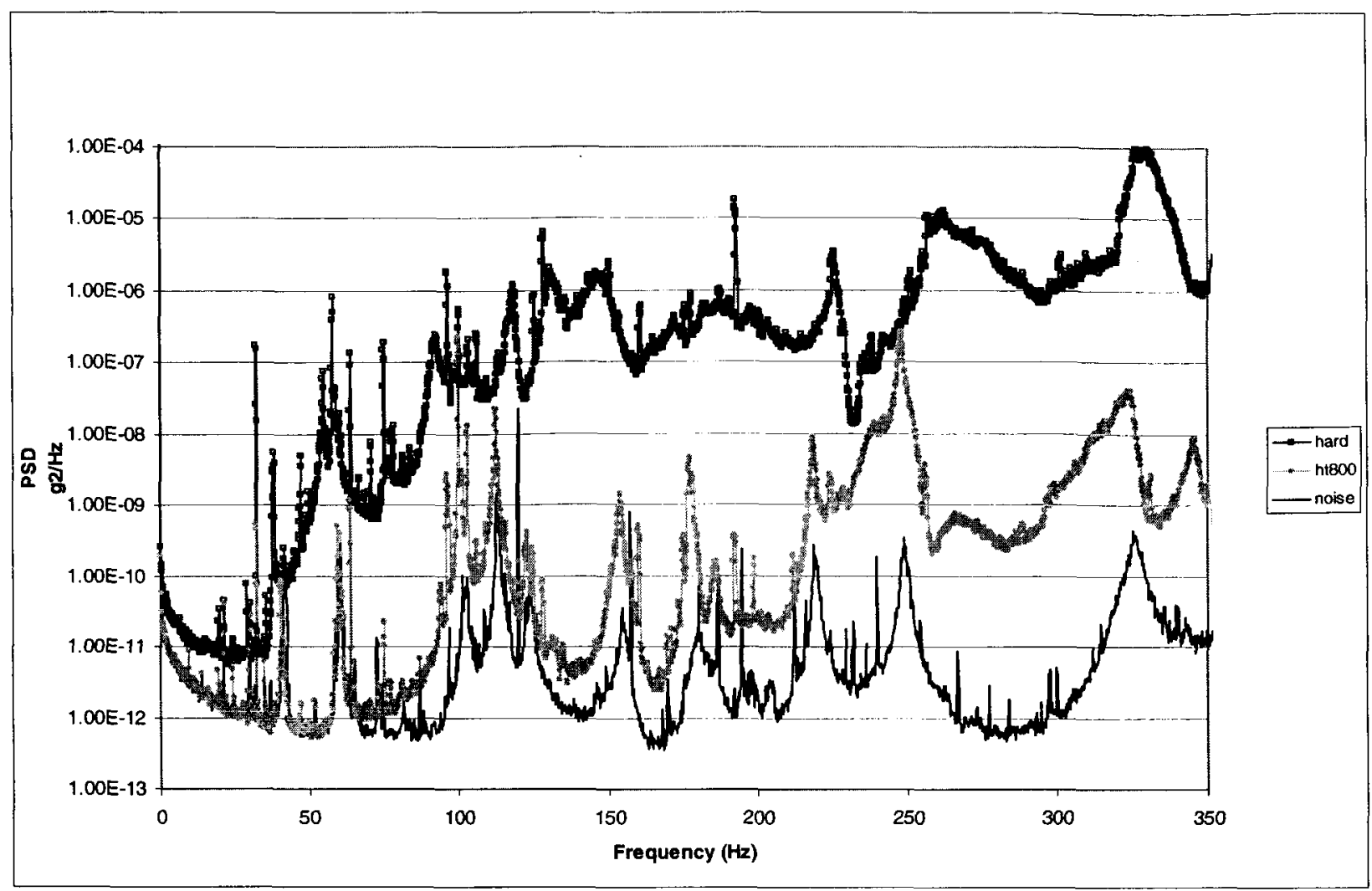

Figure 16. Performance of BISCO HT-800 compared to noise and hard shim cases at location $7 \mathrm{z}$.

\section{REFERENCES}

[1] SSP 50036, Microgravity Control Plan, Revision C, April 2003.

[2] SSP 50318, Node 3 Prime Item Description Specification, Revision C, April 2003.

[3] Bates, L., et al, "Microgravity Requirements for ISS Node 3: Emphasis on Test of Urine Processor Assembly for Microgravity Disturber Measurements", Microgravity Measurements Group Meeting 19, June 1999.

[4] Houston, J. MG-00-416 "International Space Station Node 3 Microgravity Assessment", May 2000.

[5] Houston, J. MG-02-203 "Node 3 ECLSS Racks Micro-g Analysis Status", March 2002.

[6] Houston, J. MG-02-543 "Microgravity Assessment of the Development Distillation Assembly Direct Force Transducer Data", August 2002.
[7] Holder, D. and Hutchens, C. "Development Status of the International Space Station Urine Processor Assembly", SAE \#2003-01-2690, presented at the $33^{\text {rd }}$ International Conference on Environmental Systems, Vancouver, Canada July 2003.

[8] Houston, J. MG-02-797 "Node 3 Development Distillation Assembly Microgravity Isolator Materials Characterization Test Data Report", November 2002.

\section{Acknowledgments}

Special thanks are given to Sorbothane Incorporated of Kent, Ohio, the maker of Sorbothane, and to Rogers Corporation of Elk Grove Village, Illinois, the maker of BISCO materials. Both manufacturers provided free samples of their materials for our testing, as well as technical information as was available. In addition, special recognition is due to Mr. D. Counter of MSFC for his recommendation of the HT-800 BISCO material. The efforts of MSFC Structural and Dynamics test team; particularly Mr. T. Driskill and Ms. K. Kappus, are appreciated. Finally, the authors give credit to the UPA team for their commitment to preserve the ISS microgravity environment and their willingness to modify their hardware. 